\title{
Análise da interação de surdos com um aplicativo de realidade aumentada em dispositivos vestíveis para ensino a distância de tarefas manuais
}

\author{
Thiago A. C. de Araujo', Francisco C. M. B. Oliveira1, Cecília Lis Costa Aguiar² \\ ${ }^{1}$ Universidade Estadual do Ceará (UECE) \\ Fortaleza, CE - Brasil \\ ${ }^{2}$ Universidade de Federal do Ceará (UFC) \\ Fortaleza, CE - Brasil \\ \{thiago.ac.araujo, fran.mb.oliveira, cecier.costa\}@gmail.com
}

\begin{abstract}
The so-called "quota law" facilitates access to the job market for Persons with Disabilities (PcD) in Brazil. However, many of these positions remain unfilled due to the lack of skills between PcD. We are developing courses geared towards professions that require a more practical approach, which require remotely oriented workshops. Augmented reality workshops seem to be an interesting alternative since pupils can keep their hands free by receiving instructions and interacting with their tutors who are away. This paper presents the results of an experiment with 80 people who used an augmented reality learning object with four different forms of interaction.
\end{abstract}

Resumo. A chamada "lei de cotas" facilita o acesso ao mercado de trabalho para Pessoas com Deficiência (PcD) no Brasil. No entanto, muitas dessas posições permanecem não preenchidas devido à falta de habilidades entre as PcDs. Estamos desenvolvendo cursos voltados para profissões que requerem uma abordagem mais prática, que exigem oficinas orientadas remotamente. Oficinas de realidade aumentada parecem ser uma alternativa interessante uma vez que os usuários podem manter suas mãos livres, recebendo instruções e interagindo com tutores remotos e material didático. Este artigo apresenta os resultados de um experimento com 80 pessoas que utilizaram um objeto de aprendizagem com realidade aumentada com quatro diferentes formas de interação.

\section{Introdução}

No Brasil, segundo dados do Censo 2010, realizado pelo IBGE - Instituto Brasileiro de Geografia e Estatística, existem cerca de 45 milhões de pessoas com algum tipo de deficiência, dos quais 9,7 milhões são pessoas com deficiência auditiva (IBGE, 2010).

A Lei 8213/91, chamada Lei de Cotas, determina no artigo 93 que a empresa com 100 ou mais empregados está obrigada a preencher de $2 \%$ a $5 \%$ dos seus cargos com beneficiários da previdência pública reabilitados ou Pessoas com Deficiência (PcDs) habilitadas. Apesar da legislação específica existente pode-se observar que o grande entrave da inserção e manutenção da $\mathrm{PcD}$ no mercado de trabalho está relacionada à carência na qualificação profissional e aos sistemas de habitação e reabilitação, bem como a falta de estímulos econômicos que facilitem sua contratação pelas empresas, dentre outros. A falta de metodologias e materiais didáticos adaptados para a devida 
VII Congresso Brasileiro de Informática na Educação (CBIE 2018)

Anais do XXIX Simpósio Brasileiro de Informática na Educação (SBIE 2018)

qualificação desse público alvo é perceptível. Como consequência deste problema, a PcD enfrenta dificuldades para alocação no mercado de trabalho e é subjugada a trabalhos de baixíssima produção intelectual. Para as pessoas Surdos(as) ou com Deficiência Auditiva (SDAs), público alvo desta pesquisa, este problema torna-se mais acentuado devido à barreira da comunicação.

No entanto, devido à deficiência, a educação formal é muitas vezes comprometida e dificulta o processo de aprendizagem como um todo (PINTO et al., 2015). Em resposta a isso, observa-se o desenvolvimento de uma plataforma acessível de aprendizagem à distância (web e aplicativo móvel) e oferece cursos para as PcDs. O conteúdo do curso também é acessível à medida que o sistema se adapta para transmitir conteúdo de acordo com o tipo de deficiência do aluno (linguagem de sinais, vídeo-descrição, audiodescrição, etc.) (OLIVEIRA et al, 2016). A maioria dos nossos cursos está no campo da tecnologia da informação (programação, administração de banco de dados, etc.).

O autor deste trabalhou no Le@d atuando como tutor em algumas turmas dos cursos de Introdução à Lógica de Programação e de Programação Orientada a Objetos com Java. Essa experiência permitiu perceber certa dificuldade de alguns alunos SDAs com os conceitos abstratos naturais da área de programação. Atualmente o laboratório está desenvolvendo cursos voltados para profissões que requerem uma abordagem mais prática (por exemplo, montagem de computadores, reparos eletrônicos). Tais cursos exigem oficinas orientadas remotamente.

As oficinas com uso de Realidade Aumentada (RA) e dispositivos vestíveis parecem ser uma alternativa interessante, uma vez que os usuários podem manter suas mãos livres enquanto recebem instruções e interagem com seus tutores que estão longe.

Dispositivos vestíveis como smartglasses (óculos inteligentes) ou dispositivos de realidade virtual para smartphones podem ser úteis. A investigação inicial apresenta um conjunto de perguntas de partida:

a) Até que ponto esses dispositivos podem promover acessibilidade?

b) Que tipo de interações são mais recomendadas, especialmente para SDAs em oficinas de assistência remota?

c) Como o conteúdo acessível pode ser entregue em tal configuração?

d) Por serem mais baratos, os dispositivos de realidade virtual para smartphones são capazes de fornecer uma experiência ao usuário com qualidade equivalente aos smartglasses?

\section{Trabalhos Relacionados}

No trabalho dos autores Gauglitz et al. (2012), foi desenvolvido um framework de telecolaboração móvel discreta, para permitir a um usuário interagir de maneira espacial com um ambiente remoto, através do uso de RA. O sistema é proposto de forma heterogênea, possibilitando sua aplicação a uma grande gama de dispositivos e hardwares. Foi implementado um protótipo utilizando a estrutura do framework, introduzindo diversos elementos de interface inovadores para esse tipo de ambiente. $\mathrm{O}$ sistema permite que um usuário remoto interaja fornecendo feedback através de marcações estabilizadas no mundo físico que serão visíveis a um usuário local por meio de RA. Os usuários local e remoto possuem visões desacopladas do ambiente de RA, 
VII Congresso Brasileiro de Informática na Educação (CBIE 2018)

Anais do XXIX Simpósio Brasileiro de Informática na Educação (SBIE 2018)

atualizadas em tempo real, permitindo, inclusive, que usuário remoto aponte para objetos que ainda não estão no campo de visão do usuário local.

Outra pesquisa bastante relevante é o "BeThere". O sistema foi proposto para interação entre usuário remoto e local, com um ambiente físico. Neste cenário, um usuário remoto pode conectar-se ao sistema para interagir ou auxiliar o usuário local em uma determinada tarefa através de interações 3D em um ambiente compartilhado. De acordo com o autor (Sodhi et al. (2013)), as principais vantagens do protótipo são: o compartilhamento do ambiente físico e representações tridimensionais; permitir entradas espaciais 3D, de forma a mapear naturalmente ações que seriam feitas em interações com o ambiente real, como apontar um objeto; e os usuários, remoto e local, possuem visões distintas do ambiente.

Os autores Lin e Chang (2015) desenvolveram um jogo para estimular movimentos corporais em crianças com alguma deficiência física. Foram utilizadas técnicas de visão computacional para mapear partes do corpo, permitindo às crianças interagirem fisicamente com objetos virtuais. Dessa forma são acrescentadas novas motivações para a realização das atividades físicas.

Outros trabalhos foram avaliados, tanto relacionados a colaboração remota com RA, quanto sua aplicação em educação e acessibilidade: Redondo et al. (2013), um sistema de ensino com realidade aumentada aplicado a disciplinas de arquitetura. Os experimentos executados comprovaram o aumento de desempenho dos alunos na disciplina ao usar o sistema; Barros Filho (2005), que apresenta um framework para desenvolvimento de aplicações educacionais de RA em dispositivos móveis; e por último, Imbert et al. (2013), constataram que RA tem sido amplamente utilizada nas áreas de engenharia, educação, medicina e indústria.

Estes exemplos demonstram o crescente número de aplicabilidades da tecnologia de RA. Este trabalho foca em duas dessas aplicabilidades em particular: educação e acessibilidade.

\section{Objeto de Aprendizagem com Realidade Aumentada}

\subsection{Cenário}

Métodos para atender o aluno surdo não consistem em apenas traduzir os serviços para língua de sinais, eles precisam usar recursos que facilitem o desenvolvimento das habilidades (DORIA, 1961). Segundo Dainese, Garbin e Kirner (2003), é fundamental o uso de recursos visuais para melhorar a compreensão e aprendizado de pessoas surdas.

Considerando o cenário para oferta de cursos pela plataforma EAD2PCD que propõem o ensino de habilidades manuais, acredita-se que seja necessário a criação de um novo objeto de aprendizagem para manter a atenção do aprendiz surdo no seu espaço de trabalho. Os óculos permitem que os alunos mantenham as mãos livres para serem utilizadas na realização das atividades. Técnicas de detecção de objetos no campo de trabalho juntamente com recursos de realidade aumentada possibilitam que o usuário mantenha o foco no seu espaço. Instruções e vídeos explicativos podem ser exibidos junto a área de trabalho, logo o aluno não precisa mover o seu foco de atenção para nenhum outro lugar em busca de auxílio, por exemplo. 
VII Congresso Brasileiro de Informática na Educação (CBIE 2018)

Anais do XXIX Simpósio Brasileiro de Informática na Educação (SBIE 2018)

O objeto de aprendizagem deve servir para tirar dúvidas e permitir que se façam avaliações remotas sobre as habilidades dos alunos ao realizarem tarefas relativas ao curso em questão. Os alunos gravam suas atividades e submetem os vídeos à plataforma EAD2PCD. Tutores assistem aos vídeos enviados e podem atribuir notas aos alunos. Há emissão de certificados ao final do curso para aqueles que demonstram habilidades mínimas de acordo com o plano de ensino.

Em suma, no presente contexto, este novo OA do ambiente EAD2PCD consiste no ensino de tarefas manuais a distância para PcDs, dentre elas SDA. A principal tarefa do usuário será montar um determinado objeto de acordo com as etapas predefinidas. $\mathrm{O}$ usuário poderá navegar para escolher a tarefa a ser executada. $\mathrm{O}$ aplicativo, por sua vez utiliza a imagem do campo de visão do usuário e reconhece e valida as tarefas executadas pelo usuário. Como ações fundamentais o usuário deve fazer o controle da aplicação por gestos manuais indicativos.

Porém, antes da construção deste OA, faz-se necessário fazer uma investigação para descobrir qual a combinação entre óculos e interação com o usuário, neste trabalho chamada de Conjunto de Interação (CI), oferece a melhor relação custo-benefício para a solução. É nesse ponto que se encontra a principal contribuição deste trabalho.

\subsection{Requisitos do Sistema}

Dado o cenário exposto e para atender ao objetivo deste trabalho, o aplicativo construído atende aos seguintes requisitos:

a) Projetar o objeto 3D da oficina sobre uma superfície na área de trabalho do usuário;

b) Permitir ao usuário navegar pela atividade por etapas;

c) Permitir que o usuário interaja com o sistema de forma natural;

d) Localizar e identificar peças do objeto a ser montado para ajudar o usuário;

\subsection{Interface do aplicativo}

Para o aplicativo, foi desenvolvido uma TUI. O principal recurso tangível consiste de uma mini mesa giratória que permite ao usuário visualizar o objeto 3D projetado em diversos ângulos. A Figura 1 apresenta os elementos tangíveis da interface.

A parte virtual da interface possui diversos elementos para orientar o usuário durante a execução da tarefa. Na parte superior da tela encontra-se uma barra de indicadores. Nela estão contidos, da esquerda para a direita, os elementos estáticos de um ícone em formato de relógio analógico que aparece enquanto o recurso de ajuda está ativado; a peça em 3D da etapa atual; um contador $\mathrm{X} / \mathrm{N}$ das etapas, em que $\mathrm{X}$ representa a etapa atual da atividade e $\mathrm{N}$ a quantidade total de etapas; e um contador regressivo do tempo restante para conclusão da atividade. A medida que o usuário muda a etapa da atividade, o objeto 3D vai aparecendo sobre o alvo da mesa giratória. Quando o recurso de ajuda é solicitado, uma mão 3D aponta com o dedo indicador para a peça da etapa atual. A Figura 2 apresenta os elementos virtuais da interface. 
VII Congresso Brasileiro de Informática na Educação (CBIE 2018)

Anais do XXIX Simpósio Brasileiro de Informática na Educação (SBIE 2018)

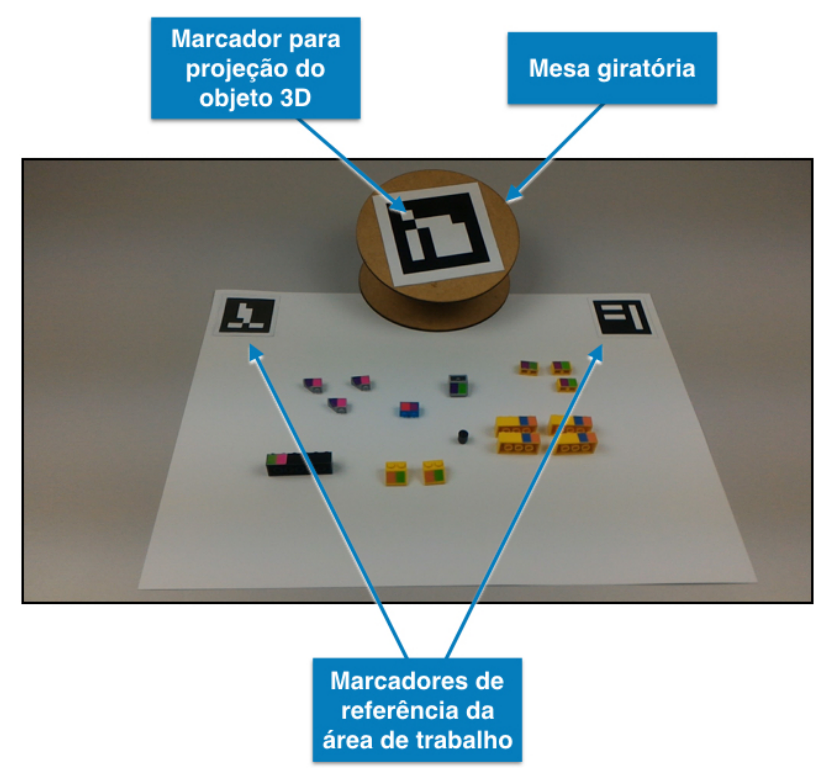

Figura 1. Elementos tangíveis da interface

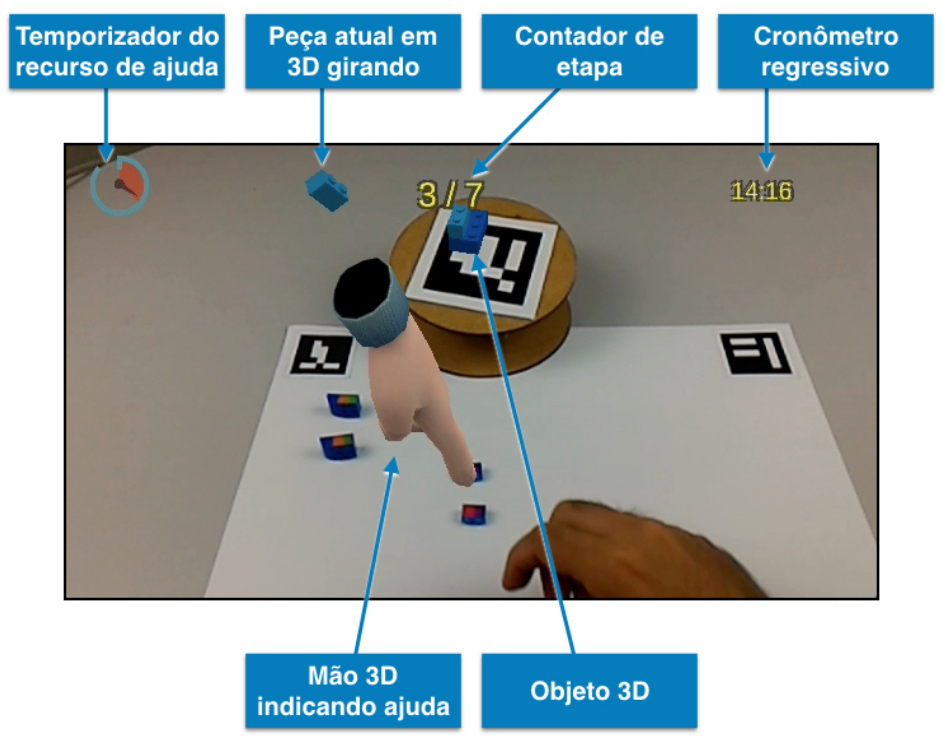

Figura 2. Elementos virtuais da interface

\subsection{Formas de interação}

\subsubsection{Acionamento de Botão Virtual}

Para a implementação dessa forma de interação optou-se por utilizar marcadores ArUco como botões para cada uma das ações possíveis (avançar, voltar e ajuda). Ao detectar o marcador, o aplicativo projeta o ícone do botão sobre o alvo. Quando o usuário oculta o marcador, um temporizador de 400ms é acionado e a cor do ícone é modificada para informar que o recurso foi devidamente acionado. Para conseguir mostrar o ícone do botão ativo enquanto o marcador está oculto, o sistema guarda a posição relativa do alvo detectado em relação ao marcador da área de trabalho localizado no canto superior 
VII Congresso Brasileiro de Informática na Educação (CBIE 2018)

Anais do XXIX Simpósio Brasileiro de Informática na Educação (SBIE 2018)

direito. A Figura 3 mostra os três botões virtuais implementados. O botão da direita está sendo ativado pelo usuário.

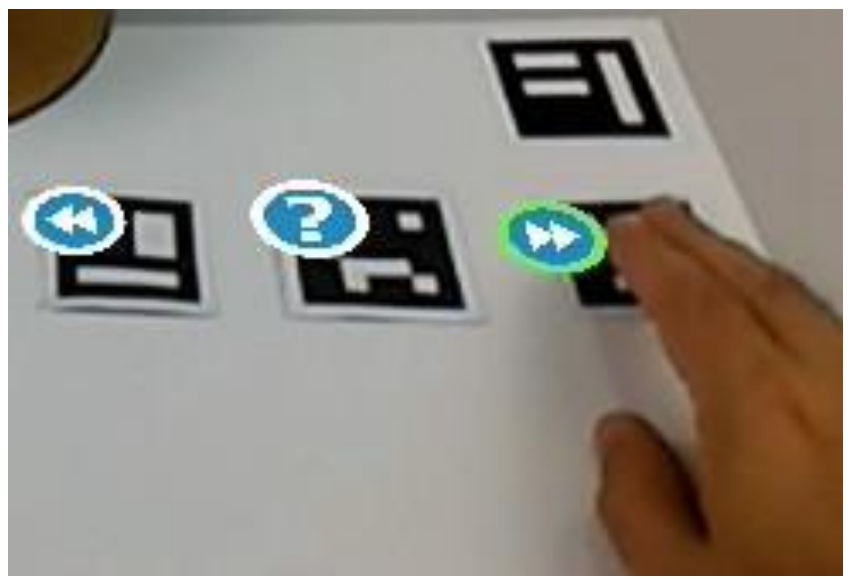

Figura 3. Botão virtual acionado

\subsubsection{Movimento dos pulsos}

Considerando o cenário de oficinas manuais, a interação natural com o sistema mais provável pode ocorrer através de gestos de mão. A solução de melhor desempenho encontrada foi o uso de marcadores nos pulsos. Como o aluno estará fazendo uma atividade de montagem, observa-se que na maior parte do tempo ele está com a palma para baixo. Desta forma, colocou-se o marcador em uma pulseira. Foi implementado uma lógica inversa ao do botão virtual. Quando o usuário desejar interagir com o sistema, ele gira o punho fazendo o marcador aparecer. Quando o marcador é visualizado pela câmera, um temporizador de $400 \mathrm{~ms}$ é acionado e uma animação no ícone de ação é feita para demonstrar para o usuário que o recurso foi corretamente acionado. Para a ação de avançar, convencionou-se mostrar o marcador na pulseira do punho direito. Para a ação de voltar, convencionou-se mostrar o marcador na pulseira do punho esquerdo. Para a ação de pedir ajuda, convencionou-se mostrar os marcadores das duas pulseiras simultaneamente. A Figura 4 mostra o o acionamento da ação de avançar com a pulseira.

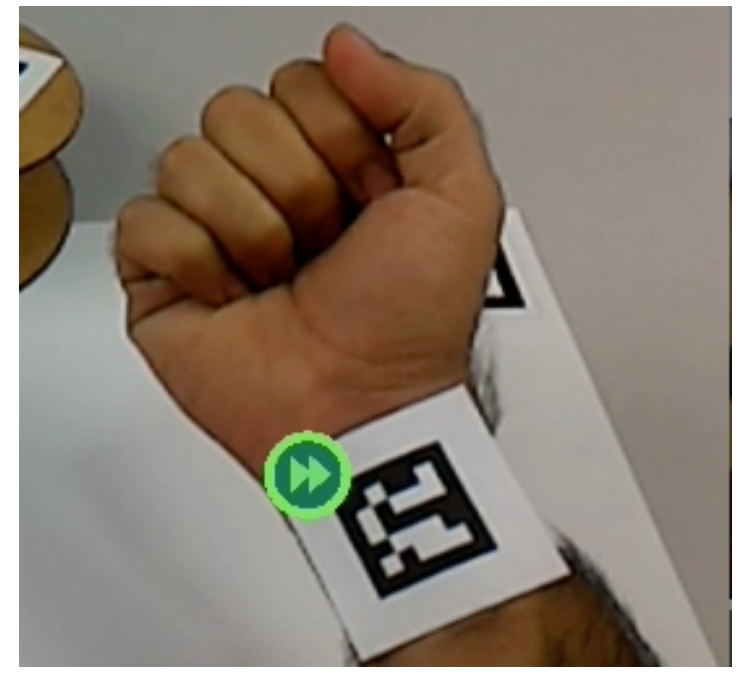

Figura 4. Acionamento da ação de avançar com a pulseira 
VII Congresso Brasileiro de Informática na Educação (CBIE 2018)

Anais do XXIX Simpósio Brasileiro de Informática na Educação (SBIE 2018)

\subsection{Dispositivos de realidade aumentada}

Além dos recursos de realidade aumentada, era de fundamental importância que o aplicativo desenvolvido funcionasse em pelo menos dois tipos de dispositivos vestíveis com suporte à realidade aumentada. Foram escolhidos o Epson Moverio BT-200, como um par de óculos inteligentes, e um dispositivo de realidade aumentada acoplado ao celular.

\section{Experimento de Validação}

Foi realizado um experimento com o objetivo de comparar a usabilidade e a experiência dos usuários surdos no desenvolvimento de uma oficina de tarefas manuais, quer usando smartglasses ou um smartphone com Cardboard. Para identificar a melhor forma de interação foi feito um teste $\mathrm{A} / \mathrm{B}$ e mensuramos a carga de trabalho utilizando o NASA Task Load Index (NASA TLX).

A atividade de e avaliação executada pelos participantes consistiam no passo-a-passo da montagem de objetos compostos por peças de Lego. O objeto de avaliação consistia de um submarino composto por 16 peças. A atividade foi as mesmas para todos os participantes.

\subsection{Conjuntos de Interação}

Foram selecionados surdos e ouvintes, como tipos de sujeito, dois dispositivos e implementadas duas formas de interação. Com isso chegou-se a oito possibilidades de CIs, conforme o Quadro 1, para utilização no experimento planejado.

\begin{tabular}{|c|c|c|c|c|c|c|}
\hline & \multicolumn{2}{|c|}{ Tipo Sujeito } & \multicolumn{2}{c|}{ Dispositivo } & \multicolumn{2}{c|}{ Interação } \\
\hline Quantidade & $\begin{array}{c}\text { Ouvinte } \\
(\text { O) }\end{array}$ & $\begin{array}{c}\text { Surdo } \\
(\text { S) }\end{array}$ & $\begin{array}{c}\text { Óculos } \\
(\text { O) }\end{array}$ & $\begin{array}{c}\text { Celular } \\
(\text { C) }\end{array}$ & $\begin{array}{c}\text { Botões } \\
(\text { B })\end{array}$ & $\begin{array}{c}\text { Pulseiras } \\
(\text { P) }\end{array}$ \\
\hline 10 & $\mathrm{X}$ & & $\mathrm{X}$ & & $\mathrm{X}$ & \\
\hline 10 & $\mathrm{X}$ & & $\mathrm{X}$ & & & $\mathrm{X}$ \\
\hline 10 & $\mathrm{X}$ & & & $\mathrm{X}$ & $\mathrm{X}$ & \\
\hline 10 & $\mathrm{X}$ & & & $\mathrm{X}$ & & $\mathrm{X}$ \\
\hline 10 & & $\mathrm{X}$ & $\mathrm{X}$ & & $\mathrm{X}$ & \\
\hline 10 & & $\mathrm{X}$ & $\mathrm{X}$ & & & $\mathrm{X}$ \\
\hline 10 & & $\mathrm{X}$ & & $\mathrm{X}$ & $\mathrm{X}$ & \\
\hline 10 & & $\mathrm{X}$ & & $\mathrm{X}$ & & $\mathrm{X}$ \\
\hline
\end{tabular}

Quadro 1. Conjuntos de Interação para o Experimento 
VII Congresso Brasileiro de Informática na Educação (CBIE 2018)

Anais do XXIX Simpósio Brasileiro de Informática na Educação (SBIE 2018)

\section{Análise dos Resultados}

Para a análise dos resultados, foi utilizado a análise de variância (ANOVA) para os fatores de tempo gasto e carga de trabalho em relação aos conjuntos de interação.

\subsection{Tempo gasto na atividade}

O tempo médio para completar a tarefa pelo conjunto $\mathrm{OCB}$ foi de 7,42 minutos, pelo conjunto SCB foi de 6,43 minutos, ambos ficando acima da média global de 4,87 minutos. A diferença entre esses dois conjuntos não é estatisticamente significante (F1,8 $=0.99, \mathrm{p}>.05)$. Mas quando comparado esses conjuntos com cada um dos 6 demais, observa-se que a diferença varia entre 1,92 e 3,60 com $p<.05$. A Figura 5 mostra o gráfico da ANOVA do tempo gasto em minutos na atividade.

As demais combinações não apresentam diferença estatística entre si. Com isso pode-se inferir que o conjunto composto por celular e interface por botões virtuais oferecem um pior desempenho quanto ao tempo, independentemente do tipo de sujeito.

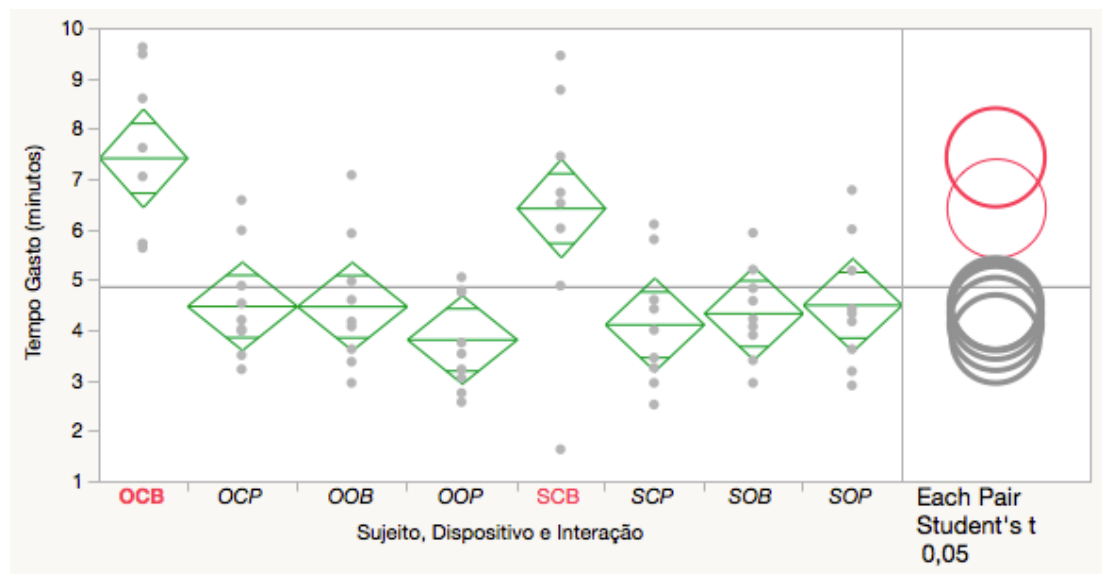

Figura 5. ANOVA Tempo gasto $(\min ) \times$ Conjunto de Interação

\subsection{Carga de trabalho na realização da atividade}

A Figura 6 mostra o gráfico da ANOVA da carga de trabalho do usuário na realização da tarefa. Observa-se que não houve diferença estatisticamente significante entre os grupos quanto a carga de trabalho. Mas percebe-se que os grupos OCB, SCB e SOB têm uma tendência de maior carga de trabalho. Isso nos permite crer que a interface de acionamentos por botões virtuais pode atrapalhar a performance do usuário. Esse fator é mais agravante para o sujeito surdo, talvez por ser uma interface que ocupa mais espaço no campo visual, pois os botões devem estar sempre visíveis na tela do dispositivo.

\section{Conclusão}

Após a análise dos resultados no cenário abordado nesta pesquisa, pode-se inferir que o tipo de interação utilizada afeta muito mais a performance do usuário na tarefa do que o tipo de dispositivo vestido. Portanto é preferível que, para a construção de um objeto de aprendizagem com uso de realidade aumentada em dispositivos vestíveis para ensino a distância de tarefas manuais, invista-se mais na melhoria da interação e nas 
VII Congresso Brasileiro de Informática na Educação (CBIE 2018)

Anais do XXIX Simpósio Brasileiro de Informática na Educação (SBIE 2018)

funcionalidades e recursos do software do que em dispositivos. Com isso é possível disponibilizar soluções de baixo custo e mais acessíveis para o público.

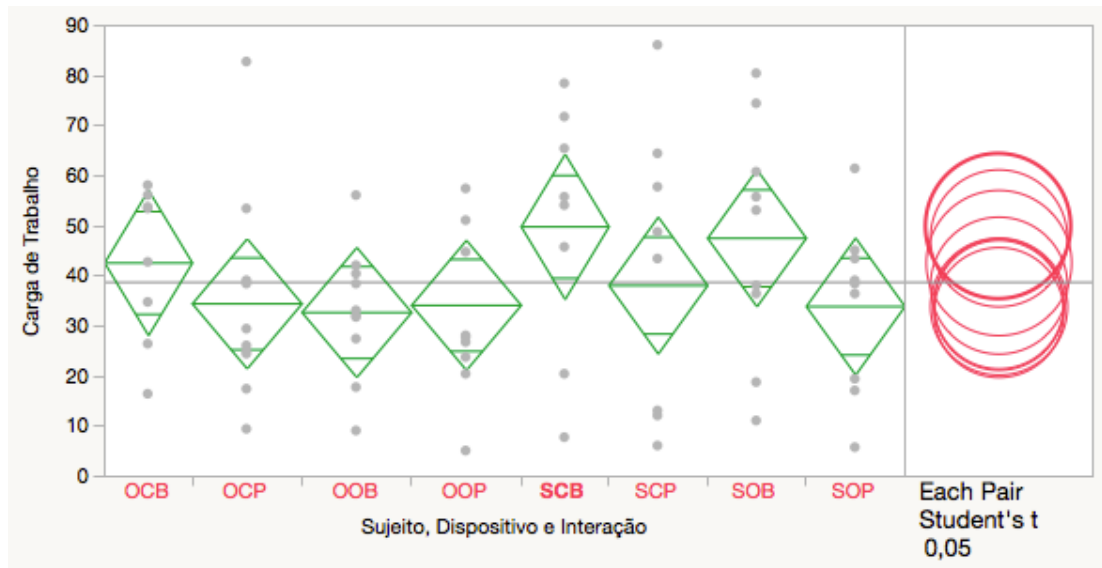

Figura 6. ANOVA Carga de trabalho x Conjunto de Interação

\section{Agradecimentos}

Agradecimentos para a Dell Computadores do Brasil, que contribuiu para o financiamento desta pesquisa.

\section{References}

Barros Filho, E. M. D. VirTraM: Um framework para o desenvolvimento de treinamentos utilizando realidade virtual em dispositivos móveis. Dissertação (Mestrado) - Universidade Federal do Ceará, 2005.

Doria, A. R. D. F. Manual de educação da criança surda. Rio de Janeiro: INES Ministério da Educação e Cultura, 1961.

Gauglitz, S.; Lee, C.; Turk, M.; Höllerer, T. Integrating the physical environment into mobile remote collaboration. In: ACM. Proceedings of the 14th international conference on Human-computer interaction with mobile devices and services. [S.1.], 2012. p. 241-250.

Imbert, N.; Vignat, F.; Kaewrat, C.; Boonbrahm, P. Adding physical properties to 3d models in augmented reality for realistic interactions experiments. Procedia Computer Science, Elsevier, v. 25, p. 364-369, 2013.

Lin, C.-Y.; Chang, Y.-M. Interactive augmented reality using scratch 2.0 to improve physical activities for children with developmental disabilities. Research in developmental disabilities, Elsevier, v. 37, p. 1-8, 2015.

Oliveira, F. C. De M. B.; Freitas, A. T. De; Araujo, T. A. C. De; Silva, L. C.; Queiroz, B. Da S.; Soares, É. F. IT education strategies for the deaf - assuring employability. In: Proceedings of the 18th International Conference on Enterprise Information Systems - Volume 2: ICEIS. [S.1.: s.n.], 2016. p. 473-482. ISBN 978-989-758-187-8. 
VII Congresso Brasileiro de Informática na Educação (CBIE 2018)

Anais do XXIX Simpósio Brasileiro de Informática na Educação (SBIE 2018)

Pinto, A. L.; Pinto, D.; Johann, J.; Johann, R. Educação de surdos: novas formas de ensinar. Caderno de Graduação-Ciências Humanas e Sociais-UNIT, v. 3, n. 1, p. 173-184, 2015.

Redondo, E.; Fonseca, D.; Sánchez, A.; Navarro, I. New strategies using handheld augmented reality and mobile learning-teaching methodologies, in architecture and building engineering degrees. Procedia Computer Science, Elsevier, v. 25, p. 52-61, 2013.

Sodhi, R. S.; Jones, B. R.; Forsyth, D.; Bailey, B. P.; Maciocci, G. Bethere: 3d mobile collaboration with spatial input. In: ACM. Proceedings of the SIGCHI Conference on Human Factors in Computing Systems. [S.1.], 2013. p. 179-188. 